\title{
A Combined View of Sterile-Neutrino Constraints from CMB and Neutrino Oscillation Measurements
}

\author{
Pawel Guzowski* \\ University of Manchester \\ E-mail: pawel.guzowski@manchester.ac.uk
}

\begin{abstract}
A light sterile neutrino that mixes with the active states has been proposed to explain anomalies in short baseline neutrino oscillation data. Constraints on the mass and mixing parameters are usually presented by showing results from complementary neutrino oscillation experiments. However, measurements of the Cosmic Microwave Background, most recently from the Planck satellite, constrain the radiative degrees of freedom in the early universe, which would be affected by a sterile neutrino. We have translated these Planck constraints into the parameter space of neutrino oscillation, that of mixing angles and mass splittings. We show these constraints from Planck compared to the muon-neutrino disappearance oscillation limits on sterile neutrinos from MINOS and IceCube, and to the recent electron-neutrino disappearance limits from reactor experiments such as Daya Bay and NEOS. We also present the cosmological limits in the context of muon-to-electron-neutrino appearance searches.
\end{abstract}

The European Physical Society Conference on High Energy Physics

5-12 July, 2017

Venice

${ }^{*}$ Speaker. 


\section{Introduction}

Anomalies in the $v_{\mu} \rightarrow v_{e}$ neutrino oscillation experiments LSND [1] and MiniBooNE [2] hint at the possibility of a light neutrino with a mass around $1 \mathrm{meV}$ that is sterile under all known Standard Model forces. These hints are in tension with other oscillation experiments, such as MINOS [3] and IceCube [4], which study $v_{\mu}$ disappearance, and Daya Bay [5] and NEOS [6], which study $v_{e}$ disappearance. The hints are also in tension with cosmological measurements, for example the Planck measurement [7] of the Cosmic Microwave Background (CMB), where the impact of additional radiative degrees of freedom in the early universe (as with a light sterile neutrino) would be seen on the power spectrum of the CMB.

In this work, we show how we can translate the cosmological limits to present them in the parameter space of the oscillation experiments. This work extends that previously published in Ref. [8], to determine the cosmological exclusion contour in the space of electron-neutrino disappearance and muon-to-electron-neutrino appearance.

\section{Method}
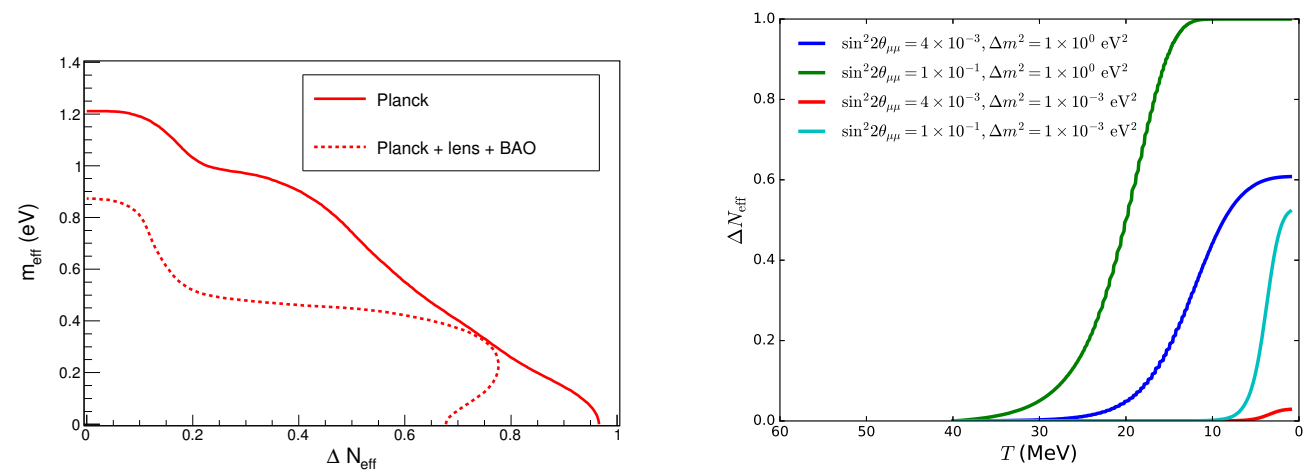

Figure 1: (Left) Planck 95\% CL exclusion limits in the cosmological parameter space. (Right) Thermalisation of a sterile neutrino from muon neutrinos in the early universe as the universe cools in temperature (T), for four different choices of parameters $\left(\sin ^{2} \theta, \Delta m^{2}\right)$.

Neutrino oscillation experiments present results in a parameter space characterised by mass splittings between neutrino mass eigenstates $\left(\Delta m_{i j}^{2}\right)$ and mixing angles $\left(\theta_{i j}\right)$ that rotate the mass eigenbasis into the flavour eigenbasis. The cosmological limits (figure 1 (left)) are made in the parameter space of additional effective radiative degrees of freedom $\left(\Delta N_{\mathrm{eff}}\right)$, and effective mass $\left(m_{\text {eff }}\right)$ which governs the temperature at which the degrees of freedom become non-radiative. We use two methods for determining the mapping between these parameter spaces, one for the case of the neutrino disappearance experiments, and one for the neutrino appearance searches.

\subsection{Neutrino disappearance}

We use the LASAGNA software toolkit [9] which solves the quantum kinetic equations [10] of neutrinos in the early universe, considering just one active neutrino mixing with one sterile neu- 
trino. We produce a mapping from oscillation parameters $\left(\sin ^{2} \theta, \Delta m^{2}\right)$ to cosmological parameters $\left(\Delta N_{\text {eff }}, m_{\text {eff }}\right)$, where $m_{\text {eff }} \approx\left(\Delta N_{\text {eff }}\right)^{\frac{3}{4}} \sqrt{\Delta m^{2}}$. An example of the thermalisation of a sterile neutrino from an early-universe temperature of $60 \mathrm{MeV}$ to $1 \mathrm{MeV}$ is shown in figure 1 (right). Once this map is produced, the calculated values of $\left(\Delta N_{\mathrm{eff}}, m_{\mathrm{eff}}\right)$ at every point in the oscillation parameter space can be compared to the Planck limit, to determine if that point is excluded by cosmological measurements. There is a slight difference between the $v_{\mu}$ and $v_{e}$ limits, due to the presence of electrons (and not muons) at the temperatures considered in the early universe.

\subsection{Neutrino appearance}

For the case of $v_{\mu} \rightarrow v_{e}$ appearance, LASAGNA is insufficient as it operates in a (1 active +1 sterile) neutrino mixing assumption, whereas the appearance search requires at least three neutrino species. We instead numerically solve the equations of Ref [11], using global fits of the 3 active neutrino mixing parameters [12], to produce the mapping in the three-dimensional parameter space $\left(\sin ^{2} \theta_{14}, \sin ^{2} \theta_{24}, \Delta m_{41}^{2}\right)$. We further reduce the two mixing angles onto a single angle $\sin ^{2} \theta_{\mu e}=\sin ^{2} 2 \theta_{14} \sin ^{2} \theta_{24}$, by selecting the least constraining value of $\Delta N_{\text {eff }}$ along contours of constant $\sin ^{2} \theta_{\mu e}$.

\section{Results}

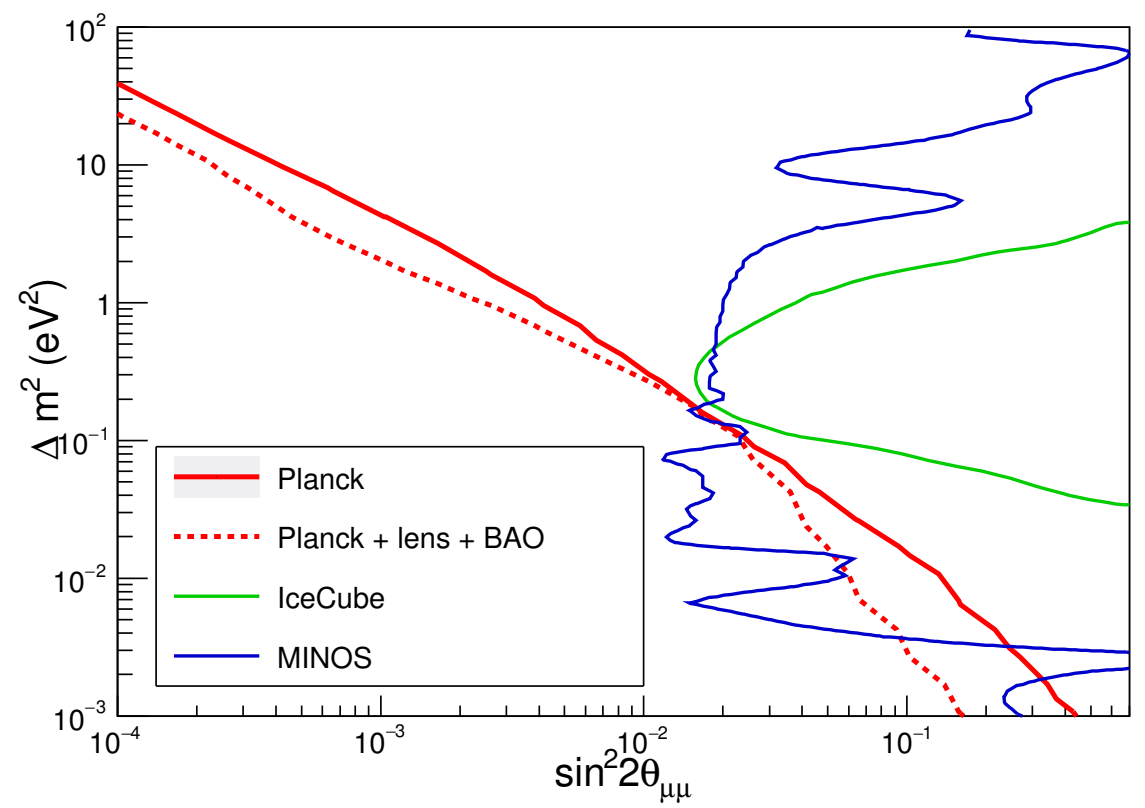

Figure 2: 95\% CL exclusion contours in the space of $v_{\mu}$ disappearance. The Planck contour (both alone, and combined with lensing and BAO data) is shown in comparison to the limits set by the oscillation experiments MINOS and IceCube. Regions to the top and/or right of the lines are excluded.

Figure 2 shows the Planck exclusion contour in the parameter space of $v_{\mu}$ disappearance. The Planck contour is shown for both Planck-alone data, and also when additional constraints 
are provided by gravitational lensing and baryon acoustic oscillation (BAO) measurements. The Planck contour excludes most of the space already excluded by oscillation experiments, with some parameter space at low values of $\Delta m^{2}$ more constrained by oscillation searches.

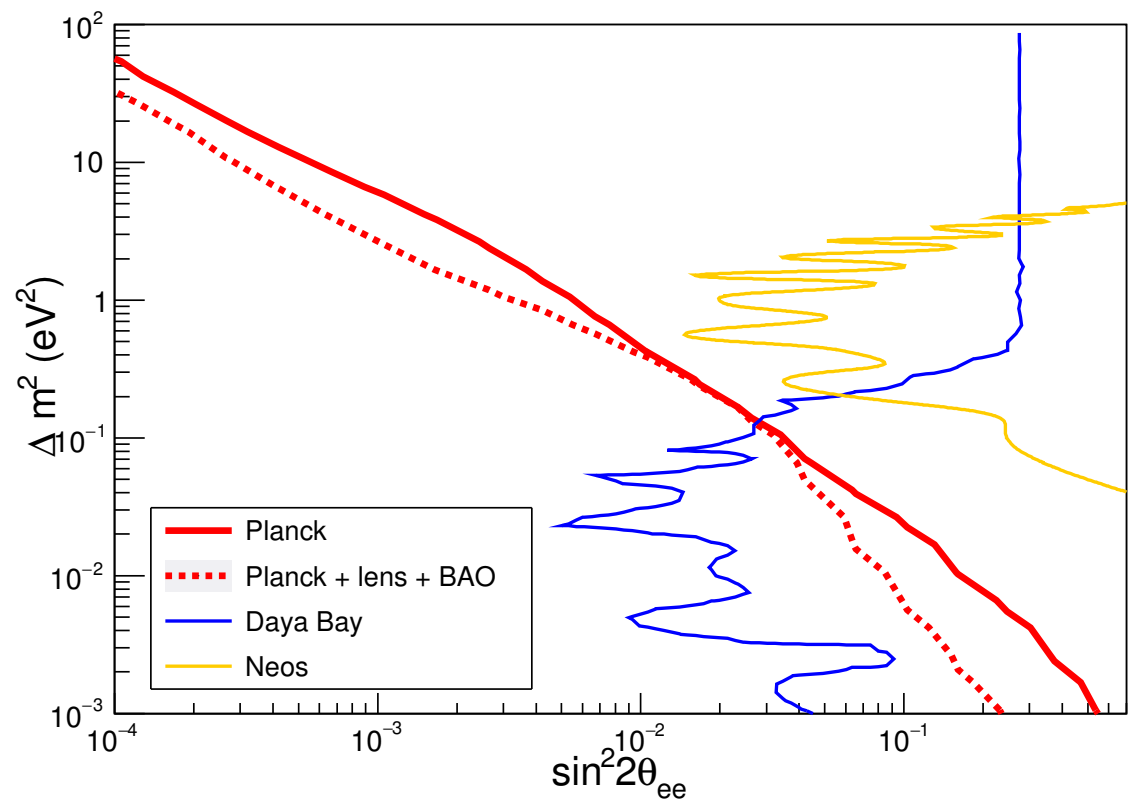

Figure 3: 95\% CL exclusion contours in the space of $v_{e}$ disappearance. The Planck contour (both alone, and combined with lensing and $\mathrm{BAO}$ data) is shown in comparison to the limits set by the oscillation experiments Daya Bay and NEOS. Regions to the top and/or right of the lines are excluded.

Similarly, figure 3 shows the Planck exclusion contour in the parameter space of $v_{e}$ disappearance, and compared to oscillation experiments sensitive to this.

Figure 4 shows the cosmological limits on the sterile neutrino in the space of $v_{\mu} \rightarrow v_{e}$ appearance, where hints have been seen. The cosmological limit excludes all of the allowed regions of LSND and MiniBooNE.

\section{Conclusion}

All three of these results show the complementarity of the cosmological limits with the oscillation searches. The oscillation experiments are usually limited by the mixing angle parameters $\left(\sin ^{2} \theta\right)$, whereas the cosmological limits provides good exclusion at low mixing angles, but only at larger mass splittings.

The cosmological limits as presented in the neutrino oscillation parameter space are modeldependent. The introduction of new phenomena that can alter the evolution of $\Delta N_{\text {eff }}$ in the early universe, for example a primordial lepton asymmetry [10], or a new pseudoscalar field that interacts with neutrinos [14], will modify the translation of the cosmological limits into the oscillation parameter space. This model-dependence could allow the LSND and MiniBooNE hints to become consistent with cosmological limits again. 


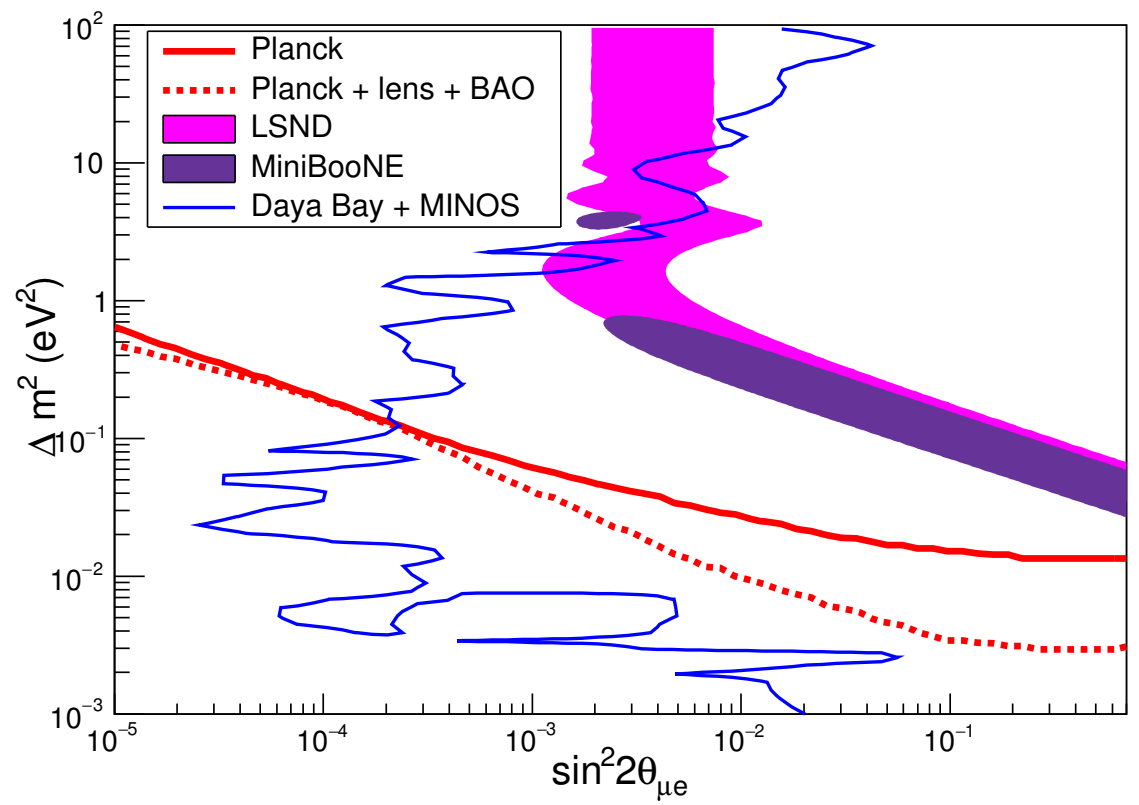

Figure 4: $95 \% \mathrm{CL}$ exclusion contours (lines) and allowed regions (filled areas) in the space of $v_{\mu} \rightarrow v_{e}$ appearance. The Planck contour (both alone, and combined with lensing and BAO data) is shown in comparison to the limit set by a combination of MINOS and Daya Bay [13], and the allowed regions observed by LSND and MiniBooNE. Regions to the top and/or right of the lines are excluded.

\section{References}

[1] A. Aguilar-Arevalo et al., Evidence for neutrino oscillations from the observation of $\bar{v}_{e}$ appearance in $a \bar{v}_{\mu}$ beam, Phys. Rev. D 64112007 (2001), https://doi.org/10.1103/PhysRevD.64.112007

[2] A. Aguilar-Arevalo et al., Search for Electron Neutrino Appearance at the $\Delta m^{2} \sim 1 \mathrm{eV}^{2}$ Scale, Phys. Rev. Lett. 98231801 (2007), https://doi.org/10.1103/PhysRevLett.98.231801

[3] P. Adamson et al., Search for Sterile Neutrinos Mixing with Muon Neutrinos in MINOS, Phys. Rev. Lett. 117151803 (2016), https://doi.org/10.1103/PhysRevLett.117.151803

[4] M. G. Aartsen et al., Searches for Sterile Neutrinos with the IceCube Detector, Phys. Rev. Lett. 117 071801 (2016), https://doi.org/10.1103/PhysRevLett.117.071801

[5] F. P. An et al., Improved Search for a Light Sterile Neutrino with the Full Configuration of the Daya Bay Experiment, Phys. Rev. Lett. 117151802 (2016), https://doi.org/10.1103/PhysRevLett.117.151802

[6] Y. J. Ko et al., Sterile Neutrino Search at the NEOS Experiment, Phys. Rev. Lett. 118121802 (2017), https://doi.org/10.1103/PhysRevLett.118.121802

[7] P. A. R. Ade et al., Planck 2015 results XIII. Cosmological parameters, Astronomy \& Astrophysics 594 A13 (2016), https://doi.org/10.1051/0004-6361/201525830

[8] S. Bridle, J. Elvin-Poole, J. Evans, S. Fernandez, P. Guzowski and S. Söldner-Rembold, A combined view of sterile-neutrino constraints from CMB and neutrino oscillation measurements, Phys. Lett. B 764322 (2016), https://doi.org/10.1016/j.physletb.2016.11.050 
[9] S. Hannestad, R. S. Hansen and T. Tram, Can active-sterile neutrino oscillations lead to chaotic behavior of the cosmological lepton asymmetry?, J. Cosmol. Astropart. Phys. 1304032 (2013), https://doi.org/10.1088/1475-7516/2013/04/032

[10] S. Hannestad, I. Tamborra and T. Tram, Thermalisation of light sterile neutrinos in the early universe, J. Cosmol. Astropart. Phys. 1207025 (2012), https://doi.org/10.1088/1475-7516/2012/07/025

[11] A. Mirizzi, N. Saviano, G. Miele and P. D. Serpico, Light sterile neutrino production in the early universe with dynamical neutrino asymmetries, Phys. Rev. D 86053009 (2012), https://doi.org/10.1103/PhysRevD.86.053009

[12] I. Esteban et al., Updated fit to three neutrino mixing: exploring the accelerator-reactor complementarity, J. High Energ. Phys. 201787 (2017), https://doi.org/10.1007/JHEP01(2017)087

[13] P. Adamson et al., Limits on Active to Sterile Neutrino Oscillations from Disappearance Searches in the MINOS, Daya Bay, and Bugey-3 Experiments, Phys. Rev. Lett. 117151801 (2016), https://doi.org/10.1103/PhysRevLett.117.151801

[14] M. Archidiacono et al., Pseudoscalar-sterile neutrino interactions: reconciling the cosmos with neutrino oscillations, J. Cosmol. Astropart. Phys. 1608067 (2016), https://doi.org/10.1088/1475-7516/2016/08/067 